\title{
Nanowire Magnetic Force Sensors Fabricated by Focused-Electron-Beam-Induced Deposition
}

\author{
H. Mattiat, ${ }^{1,2}$ N. Rossi, ${ }^{1,2}$ B. Gross, ${ }^{1,2}$ J. Pablo-Navarro $\odot,{ }^{3,4}$ C. Magén $\odot,{ }^{3,4}$ R. Badea, ${ }^{5}$ J. Berezovsky, ${ }^{5}$ \\ J. M. De Teresa $\oplus^{3,4}$ and M. Poggio $\oplus^{1,2, *}$ \\ ${ }^{1}$ Department of Physics, University of Basel, 4056 Basel, Switzerland \\ ${ }^{2}$ Swiss Nanoscience Institute, University of Basel, 4056 Basel, Switzerland \\ ${ }^{3}$ Laboratorio de Microscopías Avanzadas (LMA), Instituto de Nanociencia de Aragón (INA), Universidad de \\ Zaragoza, 50018 Zaragoza, Spain \\ ${ }^{4}$ Instituto de Ciencia de Materiales de Aragón and Departamento de Física de la Materia Condensada, \\ CSIC-Universidad de Zaragoza, 50009 Zaragoza, Spain \\ ${ }^{5}$ Department of Physics, Case Western Reserve University, Cleveland, Ohio 44106, USA
}

(Received 1 November 2019; revised manuscript received 22 January 2020; accepted 18 March 2020; published 16 April 2020)

\begin{abstract}
We demonstrate the use of individual magnetic nanowires (NWs), grown by focused-electron-beaminduced deposition (FEBID), as scanning magnetic force sensors. Measurements of their mechanical susceptibility, thermal motion, and magnetic response show that these NWs possess high-quality flexural mechanical modes and a strong remanent magnetization pointing along their long axis. Together, these properties make the NWs excellent sensors of weak magnetic field patterns, as confirmed by calibration measurements on a micron-sized current-carrying wire and magnetic scanning-probe images of a permalloy disk. The flexibility of FEBID in terms of the composition, geometry, and growth location of the resulting NWs, makes it ideal for fabricating scanning probes specifically designed for imaging subtle patterns of magnetization or current density.
\end{abstract}

DOI: 10.1103/PhysRevApplied.13.044043

\section{INTRODUCTION}

In the early 1800 s, images of the stray magnetic fields around permanent magnets and current-carrying wires made with tiny iron filings played a crucial role in the development of the theory of electromagnetism. Today, magnetic imaging techniques such as Lorentz microscopy, electron holography, and a number of scanning-probe microscopies continue to provide invaluable insights. Images of magnetic skyrmion configurations [1] or of edge and surface currents in topological insulators [2] have provided crucial direct evidence for these phenomena. The ability to map magnetic field sensitively and on the nanometer-scale - unlike global magnetization or transport measurements - overcomes ensemble or spatial inhomogeneity in systems ranging from arrays of nanometer-scale magnets, to superconducting thin films, to strongly correlated states in van der Waals heterostructures. Local imaging of nanometer-scale magnetization [3], local Meissner currents [4], or current in edge states [5] is the key to unraveling the microscopic mechanisms behind a wealth of new and poorly understood condensedmatter phenomena.

\footnotetext{
*martino.poggio@unibas.ch
}

The techniques combining the highest magnetic field sensitivity with the highest spatial resolution include scanning Hall-bar microscopy, scanning nitrogen-vacancy $(\mathrm{N}-V)$ center magnetometry, and scanning superconducting quantum interference device (SQUID) microscopy. Each has demonstrated a spatial resolution better than $100 \mathrm{~nm}$ and a magnetic field sensitivity ranging from $500 \mu \mathrm{T} / \sqrt{\mathrm{Hz}}$ for Hall-bar microscopy [6], to $60 \mathrm{nT} / \sqrt{\mathrm{Hz}}$ for $\mathrm{N}-V$ magnetometry [7], and $5 \mathrm{nT} / \sqrt{\mathrm{Hz}}$ for scanning SQUID microscopy [8]. Recently, a form of magnetic force microscopy (MFM) based on a transducer made from a magnet-tipped nanowire (NW) demonstrated a high sensitivity to magnetic field gradients of $11 \mathrm{mT} /(\mathrm{m} \sqrt{\mathrm{Hz}})$ with a similar spatial resolution [9]. The high force sensitivity of NW cantilevers coupled together with a small magnetic tip size could allow such sensors to work both close to a sample, maximizing spatial resolution, and in a regime of weak interaction, remaining noninvasive.

Here, we demonstrate the use of individual magnetic NWs, patterned by focused-electron-beam-induced deposition (FEBID), as MFM transducers for mapping magnetic fields with high sensitivity and resolution. The monopolelike magnetic charge distribution of their tips makes these transducers directly sensitive to magnetic fields rather than to field gradient, as in the initial 
demonstration of NW MFM [9]. Furthermore, the FEBID fabrication process allows for a large degree of flexibility in terms of the geometry, composition, and location of the NW transducers. In particular, the possibility of long, thin, and sharp NWs is promising for further increasing field sensitivity and spatial resolution of the technique [10].

\section{FEBID NWs}

FEBID is an additive-lithography technique where precursor gas molecules are adsorbed onto a surface and dissociated by a focused electron beam, forming a local deposit [11-15]. It can be used to pattern exceptionally small features, down to a few nanometers. This highresolution patterning is complemented by the capability to produce three-dimensional structures, as well as to pattern on unconventional nonplanar surfaces, such as high-aspect-ratio tips. FEBID and its sister technique, focused-ion-beam-induced deposition (FIBID), have been used to produce deposits of various materials with metallic [16], magnetic [17,18], superconducting [19], or photonic [20] functionalities. They have been used in industry and research for mask repair [21], circuit editing, lamella fabrication [22], tip functionalization [23], and for the fabrication of nanosensors [24]. They have also been employed in the production of freestanding NWs from both superconducting [25] and - as in this work-magnetic materials $[18,26]$.

We grow freestanding NWs by FEBID using $\mathrm{Co}_{2}(\mathrm{CO})_{8}$ as a gas precursor at specific positions along the cleaved edge of a Au-coated GaAs chip. Their lengths range from 9.1 to $11.0 \mu \mathrm{m}$ and their base diameters from 105 to $120 \mathrm{~nm}$ as inferred from SEM images. In general, NWs with diameters ranging from $35 \mathrm{~nm}$ to $1 \mu \mathrm{m}$ can be grown using our FEBID technique. The NWs studied here consist of nanocrystalline $\mathrm{Co}$, with a composition reaching up to $80 \%$ [26], and residues of $\mathrm{C}$ and $\mathrm{O}$. Their proximity to the edge of the chip allows optical access from the side for the detection of their flexural motion. A SEM image of a Co NW standing at the chip edge is shown in Fig. 1(a). Surface roughness and geometric irregularities are part of the FEBID fabrication process and are present across the 11 NWs studied in this work.

\section{MEASUREMENT SETUP}

We mount the chip with as-grown Co NWs in a custom-built scanning-probe microscope, enclosed in a high-vacuum chamber at a pressure of $1 \times 10^{-6}$ mbar. The microscope includes a piezoelectric translation stage, with which we position the NW of interest into the focal spot of fiber-coupled optical interferometer for the detection of the NW's flexural motion [27]. We use a second (a)

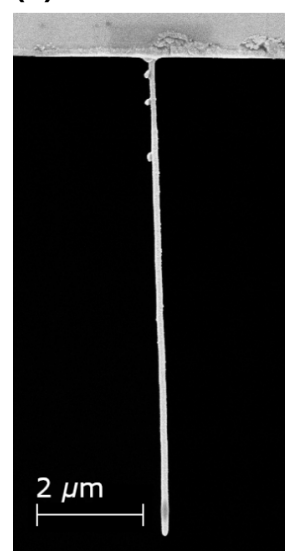

(b)

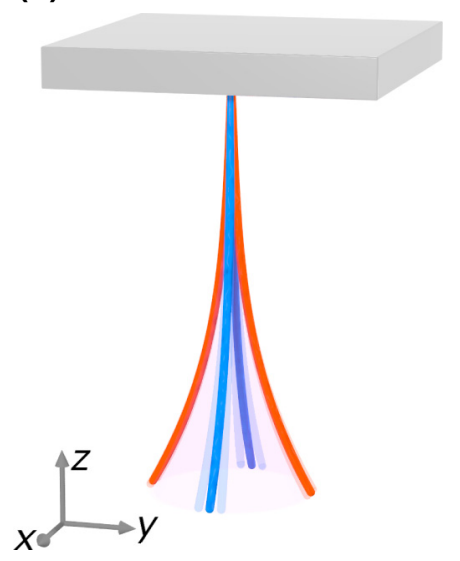

(c)

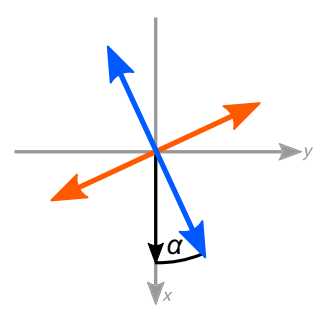

(d)

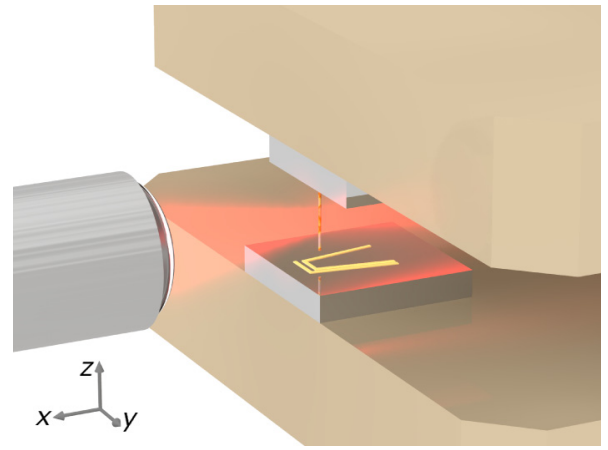

(e)

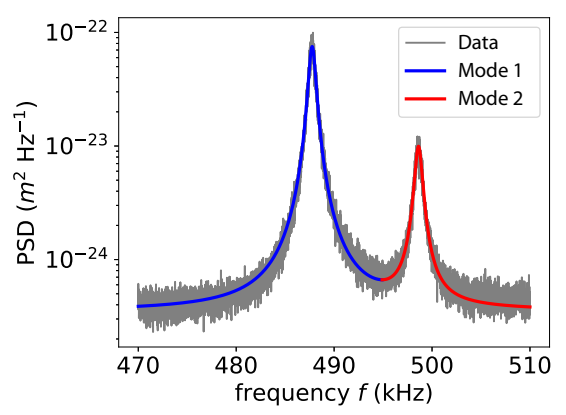

FIG. 1. (a) SEM micrograph of a Co FEBID NW at the chip edge. (b) Illustration of the NW's flexural modes. The displacement amplitudes are exaggerated for better visibility. (c) Projection of flexural modes onto the $x-y$ plane. $\alpha$ is the angle between the lower-frequency mode direction (blue) and the measurement vector (black). (d) Illustration of the optical readout of the NW motion. (e) Thermally excited response of the upper and lower mode at room temperature (gray). We fit the thermal noise displacement PSD with Eq. (A1). The two modes are highlighted by the blue and red regions of the fit. 
piezoelectric translation stage to approach and scan the sample of interest below the NW's free end, as illustrated in Fig. 1(d). This combined apparatus allows us to use individual NWs as scanning probes operating in the pendulum geometry, i.e., with their long axes perpendicular to the sample surface to prevent snapping into contact $[9,28]$.

The fiber-coupled optical interferometer operates at $1550 \mathrm{~nm}$ and provides a calibrated measurement of the NWs flexural motion projected along the measurement axis (see Appendix A). Figure 1(e) shows a typical power spectral density (PSD) of an individual NW's thermally excited flexural motion at room temperature, revealing a splitting in the resonance frequency of the fundamental mode. This well-known splitting is observed for all examined NWs and is a signature of two nearly degenerate, orthogonal flexural eigenmodes, resulting from crosssectional asymmetries and/or nonisotropic clamping [29]. The NW's coupling to the thermal bath results in a Langevin force that drives each mode equally. The difference in the amplitude of the two thermal noise peaks in Fig. 1(e) is a consequence of the projection of the NW's flexural motion onto a single measurement axis, corresponding to the direction of the optical gradient, at an angle $\alpha$ with respect to mode 1 .

\section{MECHANICAL PROPERTIES}

Measurements of the NWs' thermomechanical noise PSD are performed with the bath held at room temperature $(293 \mathrm{~K})$, liquid-nitrogen temperature $(77 \mathrm{~K})$, and liquidhelium temperature $(4.2 \mathrm{~K})$. Heating caused by absorption of the incident laser light (typically $25 \mu \mathrm{W}$ ) can increase the NW's temperature well above the bath temperature. As a result, care must be taken interpreting PSDs, as discussed in Appendix C. Using the fits to the measured PSDs based on the fluctuation-dissipation theorem, we determine the mechanical properties of the fundamental flexural modes: their resonance frequencies $f_{i}=\omega_{i} / 2 \pi$, quality factors $Q_{i}$ $(i=1,2)$, and effective motional mass $m_{\text {eff }}$ (see Appendix A) [9]. At $293 \mathrm{~K}$, the resonance frequencies of the NWs are between 390 and $560 \mathrm{kHz}$ with a mode splitting from 10 to $42 \mathrm{kHz}$. We measure quality factors around 600 and motional masses in the 100s of fg range. These parameters correspond to flexural modes with effective spring constants $k_{i}$ of a few $\mathrm{mN} / \mathrm{m}$. At a bath temperature $T_{\text {bath }}=$ $4.2 \mathrm{~K}$, the quality factors improve by roughly a factor of 3 to around 1000 to 2000 and the resonance frequencies shift upwards by roughly $30 \mathrm{kHz}$. From these parameters, shown in Table I for two different NWs, we deduce the spring constants, mechanical dissipation, and thermally limited force sensitivities. Notably, at $T_{\mathrm{NW}}=4.2 \mathrm{~K}$, a typical NW has flexural modes with thermally limited force sensitivities around $10 \mathrm{aN} / \sqrt{\mathrm{Hz}}$. In practice the force sensitivity is limited to about $25 \mathrm{aA} / \sqrt{\mathrm{Hz}}$, since even at very low laser power $(1 \mu \mathrm{W}$ on $\mathrm{NW} 4$, signal-to-noise ratio of first mode $\mathrm{SNR} \approx 8$ ) bolometric heating is present and leads to a NW temperature $T_{\mathrm{NW}} \approx 20 \mathrm{~K}$ (see Appendix C).

\section{MAGNETIC PROPERTIES}

We probe the magnetic properties of each NW by measuring its mechanical response to a uniform magnetic field $B$ up to $8 \mathrm{~T}$ applied along its long axis. In particular, we measure the shift in the resonance frequency of each flexural mode, $\Delta f_{i}=f_{i}-f_{0_{i}}$, as a function of $B$, where $f_{0_{i}}$ is the resonance frequency at $B=0$. Figure 2 shows a typical measurement of the hysteretic response of $\Delta f_{1}(B)$ and $\Delta f_{2}(B)$ carried out on NW 4 with $T_{\text {bath }}=4.2 \mathrm{~K}$. As in measurements of the other NWs, the data show a smooth $\mathrm{V}$-shaped response for most of the field range, except for discontinuous inversions of the slope ("jumps") in reverse fields of around $\pm 40 \mathrm{mT}$. These sharp features, which arise from the switching of the NW magnetization, and the steady stiffening of the mechanical response as $|B|$ increases are characteristic of a strong magnet with a square magnetization hysteresis, whose easy axis is nearly parallel to the applied field [30], as opposed to a weak magnet with comparatively small switching field and saturation magnetization. Therefore, the data point to NWs with negligible magnetocrystalline anisotropy and an easy axis coincident with their long axis, as set by the magnetic shape anisotropy resulting from their extreme aspect ratio.

In order to extract specific magnetic properties from our measurements, we compare them to micromagnetic

TABLE I. Mechanical properties of representative NWs with the thermal bath held at room temperature $\left(T_{\text {bath }}=293 \mathrm{~K}\right)$ and liquidhelium temperature $\left(T_{\text {bath }}=4.2 \mathrm{~K}\right)$ measured at high and low laser powers. Resonance frequencies, $Q$ factors, effective mass, and measurement angle (not shown here) are extracted by fitting the thermal noise PSD (see Appendix A). For a correct interpretation, bolometric heating due to the readout laser must be taken into account. Considering this effect, we also specify a NW temperature $T_{\mathrm{NW}}$ (see Appendix C). The data of the last two lines in a liquid-helium environment correspond to NW temperatures of $99 \mathrm{~K}(20 \mu \mathrm{W})$ and $22 \mathrm{~K}(1.2 \mu \mathrm{W})$, respectively.

\begin{tabular}{lccccccc}
\hline \hline$T_{\text {bath }}$ & $\mathrm{NW}$ & $f_{1}(\mathrm{kHz})$ & $f_{2}(\mathrm{kHz})$ & $\Delta f_{m}(\mathrm{kHz})$ & $Q_{1}$ & $Q_{2}$ & $m_{\text {eff }}\left(10^{-15} \mathrm{~kg}\right)$ \\
\hline 293 & 1 & 390.726 & 426.018 & 35.292 & $528.0(1.4)$ & $\ldots$ & $0.69(4)$ \\
& 4 & 514.459 & 556.750 & 42.291 & $551.1(1.5)$ & $527(66)$ & $0.260(15)$ \\
4.2 & 4 & 550.803 & 593.745 & 42.942 & $1045(1.0)$ & $1156(40)$ & $0.260(15)$ \\
& 4 & 555.860 & $599.044(49)$ & 43.18 & $2356(39)$ & $\ldots$ & $0.260(15)$ \\
\hline \hline
\end{tabular}




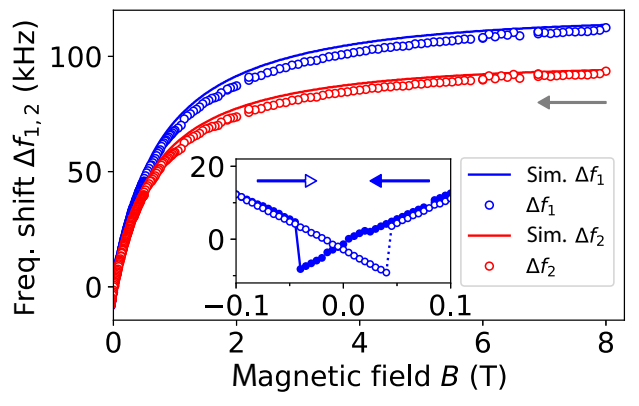

FIG. 2. Resonance frequency shift of the two first-order flexural modes of NW 4, measured while sweeping an axially aligned external magnetic field from 8 to $0 \mathrm{~T}$ (gray arrow). Points represent measured data, while solid lines correspond to simulations using Mumax3 (see Appendix B), yielding a saturation magnetization of $M_{\mathrm{sat}}=1.1(1) \times 10^{6} \mathrm{~A} \mathrm{~m}^{-1}$, which equates to $\mu_{0} M_{\text {sat }}=1.43(12) \mathrm{T}$. The inset shows a close-up plot of mode 1 's frequency shift in the switching region around zero field for increasing and decreasing sweeps of the field (blue arrows). The jumps are highlighted by solid and dashed blue lines.

simulations, which model both the NW's magnetic state and the way in which its interaction with $B$ affects the mechanical rigidity of the flexural modes. We use Mumax3 [31,32], which employs the Landau-LifshitzGilbert micromagnetic formalism with finite-difference discretization, together with geometrical and material parameters to model each NW. For a given value of $B$ in a hysteresis loop, the numerical simulation yields the equilibrium magnetization configuration and the total magnetic energy $E_{m}$ corresponding to that configuration. Just as in dynamic cantilever magnetometry (DCM) [30,33], the frequency shift of each flexural mode is proportional to the curvature of the system's magnetic energy $E_{m}$ with respect to rotations $\theta_{i}$ corresponding to each mode's oscillation:

$$
\Delta f_{i}=\frac{f_{0_{i}}}{2 k_{i} l_{e}^{2}}\left(\left.\frac{\partial^{2} E_{m}}{\partial \theta_{i}^{2}}\right|_{\theta_{i}=0}\right),
$$

where $l_{e}$ is an effective length, which takes into account the shape of the flexural mode [34]. Therefore, by numerically calculating the second derivatives of $E_{m}$ with respect to $\theta_{i}$ at each $B$, we simulate $\Delta f_{i}(B)$. Note that, unlike in standard DCM, where the magnetic sample is attached to the end of the cantilever, each NW is magnetic along its full length. Because of the mode shape, each longitudinal segment of the NW rotates by a different angle during a flexural oscillation, experiencing a different tilt of the external magnetic field. We account for this effect by applying a spatially dependent external field in the simulation, rather than altering the geometry, which is impractical. For positive (negative) deflections in experiment, the tilt direction of the field in the simulations increases (decreases) with the $z$ position along the NW. The magnitude of the tilt angle follows the Euler-Bernoulli equation, reflecting the mode shape. We choose a maximum oscillation amplitude (at the tip) large enough to account for the finite precision of the simulation. The torque signal can then be calculated using a finite-difference approximation for the second derivative in Eq. (1) and the simulated magnetic energy of the system for small positive, negative, and no deflection (see Appendix B) [33].

The excellent agreement between the measured and simulated $\Delta f_{i}(B)$ in Fig. 2 is typical for all measured NWs. For each NW, the mechanical parameters used in the simulation are extracted from measurements of the thermal motion at $B=0$, while geometrical parameters are estimated from SEM images. We adjust the value of the saturation magnetization $M_{\text {sat }}$ in order to bring the curves into agreement, giving us a sensitive measurement of this material property. $M_{\text {sat }}$ is found to be 1.1(1) $\times 10^{6} \mathrm{~A} \mathrm{~m}^{-1}$, where the uncertainty is dominated by the estimation of the NW geometry from SEM. While the saturation magnetization of bulk Co is $1.4 \times 10^{-6} \mathrm{~A} \mathrm{~m}^{-1}$, the expected metallic composition of as-grown Co NWs grown by FEBID varies between 70 and $95 \%$. This reduced Co content makes our measured value consistent with expectations as does a comparison with electron holography measurements in similar NWs, yielding $M_{\text {sat }} \simeq 1.2 \times 10^{-6} \mathrm{~A} \mathrm{~m}^{-1}$ [26]. In addition, the simulations show that the magnetization of the NWs is axially aligned in remanence for up to about $40 \mathrm{mT}$ of reverse field. These results are consistent with nanoSQUID measurements of similar Co NWs carried out at $15 \mathrm{~K}$ by Martínez-Pérez et al. [35]. This axially aligned remanent magnetization can be represented by a magnetic

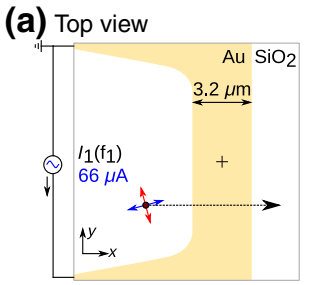

(b)

Side view

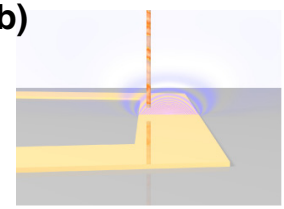

(c)

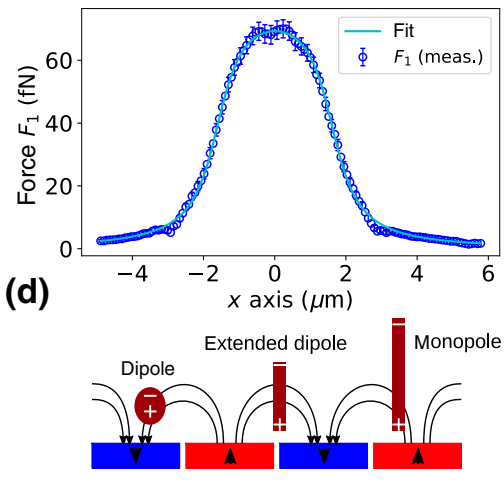

FIG. 3. (a) Sketch of the Au wire dimensions, NW mode directions, scanning direction, and applied drive tone. (b) Illustration of the scanning-probe measurement. (c) Measured response of the first mode of NW 4 as a function of position along the line scan indicated by the arrow shown in (a) to a resonant drive field for a tip-sample distance of $d_{z}=200 \mathrm{~nm}$. The best fit according to Eq. (2) is represented by the solid cyan line and yields a value of $q_{0}=9.7(4) \times 10^{-9} \mathrm{~A} \mathrm{~m}$. (d) Illustration of three tip models for magnetic tips. The FEBID NWs correspond to the monopole tip model. Figure adapted from Ref. [36]. 
charge distribution in the form of an elongated dipole, leaving a monopolelike distribution localized at the free end of the NW to interact with an underlying sample, as shown in Fig. 3(d).

\section{MEASUREMENT OF MAGNETIC FIELD PROFILE}

In order to determine the behavior of the FEBID NWs as scanning probes, we approach and scan a nearly planar sample with respect to NW 4 at $T_{\text {bath }}=4.2 \mathrm{~K}$. The sample consists of a 6- $\mu \mathrm{m}$-long, 3.2- $\mu \mathrm{m}$-wide, and 240 -nm-thick $\mathrm{Au}$ wire patterned between two contact pads on a Si substrate [Figs. 3(a) and 3(b)]. By passing a current through the wire, we produce a well-known magnetic field profile $\mathbf{B}_{\mathrm{ac}}(x, y, z)$ given by the Biot-Savart relation, with which we drive NW oscillations and calibrate its response, as done in standard MFM [37-39]. By applying an excitation current containing two sine waves, each at the frequency of one of the NW modes $f_{1}$ and $f_{2}$, we drive the NW as we scan it across the Au wire at a fixed tip-sample spacing. Both the resonance frequencies $f_{i}$ and oscillation amplitudes $r_{i}$ are tracked using two phase-locked loops. The corresponding values of the force driving each mode on resonance are calculated using $F_{i}=r_{i} k_{i} / Q_{i}$ (see Appendix D). Figure 3(c) shows the response of mode 1 to a drive current amplitude of $47 \mu \mathrm{A}$ as we scan the NW, which in the absence of a static external field $(B=0)$ is in a remanent magnetization configuration, above the Au wire at a fixed distance of $d_{z}=200 \mathrm{~nm}$. Since the first mode is nearly aligned with the $x$ direction $\left(\alpha \approx 7.3^{\circ}\right)$ and thus along the direction of $\mathbf{B}_{\mathrm{ac}}$, the orthogonal second mode has almost no response to the driving tone at $f_{2}$ and is not shown.

From our torque magnetometry measurements, we know that the magnetic NWs have an axially aligned remanent magnetization. Because the decay length of the magnetic field from our sample is much shorter than the NW length, the sample fields only interact with the monopolelike magnetic charge distribution at the free end of the NW [36]. This charge distribution then determines the NW's response to magnetic field profiles produced by a sample. For a monopolelike NW tip, we can relate the driving magnetic field and the force it produces on the NW by

$$
F_{i}=q_{0} \mathbf{B}_{\mathrm{ac}} \cdot \hat{\mathbf{r}}_{i},
$$

where $q_{0}$ is an effective magnetic monopole moment describing the tip magnetization and $\hat{r}_{i}$ is the unit vector in the direction of displacement of mode $i$. In this pointprobe approximation, we consider the interaction of dipole and higher multipoles of the magnetic charge with the driving field to be negligible. As shown by the agreement between the field calculated from the Biot-Savart law and the measured response of NW 4 in Fig. 3(c), this approximation is valid for our NWs. Control experiments, using the applied magnetic field to initialize the NW magnetization along the opposite direction also show that spurious electrostatic driving of the NW modes is negligible. Combining measurements at different $d_{z}$ and different driving currents, we find that NW 4 has an effective magnetic charge of $q_{0}=9.7(4) \times 10^{-9}$ A m. Given our thermally limited force sensitivity of $25 \mathrm{aN} / \sqrt{\mathrm{Hz}}$ at $T_{\text {bath }}=4.2 \mathrm{~K}$, this value of $q_{0}$ gives our sensors a sensitivity to magnetic field of around $3 \mathrm{nT} / \sqrt{\mathrm{Hz}}$. This sensitivity is similar to those of some of the most sensitive scanning probes available, including scanning $\mathrm{N}-V$ magnetometers and scanning SQUIDs.

The value extracted for $q_{0}$ is consistent with $M_{\text {sat }}$ found in Sec. V and the geometry of the NW. Assuming a perfect cylinder with magnetization $M_{\text {sat }}$, the expected magnetic charge is $q_{0}^{\text {cyl }}=M_{\text {sat }} \pi(d / 2)^{2}$, where $d$ is the cylinder's diameter. Given that the diameters of the NWs range from 105 to $120 \mathrm{~nm}$ we find $q_{0}^{\text {cyl }}=9.5 \times 10^{-9}$ to $12.4 \times$ $10^{-9} \mathrm{Am}$.

Furthermore, the magnetic charge model allows us to estimate the stray field and field gradients produced by the NW tip, so that we can assess its potential for perturbing the magnetic state of the sample below. At a distance of $d_{z}=50 \mathrm{~nm}$ from the NW tip, the stray magnetic field and magnetic field gradients are $B_{\text {tip }}=\mu_{0} q_{0} /\left(4 \pi z_{\text {mono }}^{2}\right) \approx$ $60 \mathrm{mT}$ and $d B_{\text {tip }} / d z \approx 1 \mathrm{MT} \mathrm{m}^{-1}$. The stray field is of similar size to that produced by a conventional MFM tip [40]. For future NW devices to be less invasive, i.e., having less magnetic charge at their tips, sharper tips than those produced here, which are more than $100 \mathrm{~nm}$ in diameter, will be required [41]. The large magnetic field gradients, however, combined with the NWs' excellent force sensitivity may make the NWs well-suited as transducers in sensitive magnetic resonance force microscopy [42].

\section{MAGNETIC FIELD IMAGING}

In Fig. 4, we demonstrate the ability to image submicrometer features using a FEBID NW as MFM sensor on a permalloy disk $\left(\mathrm{Ni}_{0.81} \mathrm{Fe}_{0.19}\right)$ at $T_{\text {bath }}=4.2 \mathrm{~K}$. Three disks, $1 \mu \mathrm{m}$ in diameter and 40-nm-thick, are patterned on top of the Au wire, of which one is imaged. During the scan, the first flexural mode of the NW is electrically driven on resonance using an ac voltage applied between the Au wire and a third lead [Fig. 4(a)]. Frequency shift and dissipation are recorded using a phase-locked loop. Figures 4(c) and 4(d) show a $1.5 \times 1.5 \mu \mathrm{m}^{2}$ image of frequency shift $\Delta f_{1}(x, y)$, as the disk is scanned below NW 4 for $d_{z}=100 \mathrm{~nm}$ at $B=0$ and for $d_{z}=150 \mathrm{~nm}$ at $B=100 \mathrm{mT}$, respectively. At $B=0$ [Fig. 4(c)], the magnetization of the disk is arranged in a remanent vortex configuration [43], as verified by a micromagnetic simulation carried out with Mumax3. Figure 4(e) shows the magnetic image contrast expected from the simulation using the monopole model (2), in which the frequency 
(a)

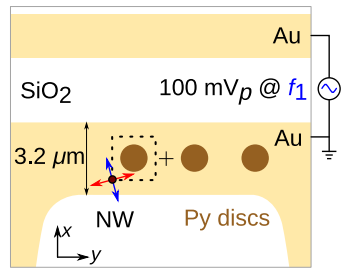

(c)

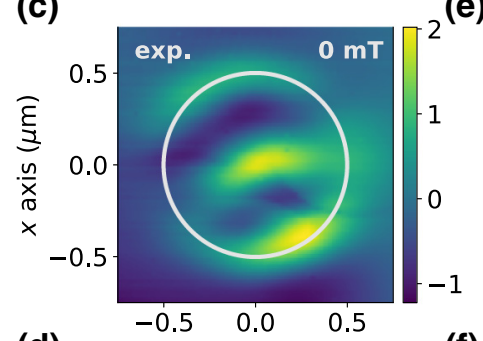

(d)

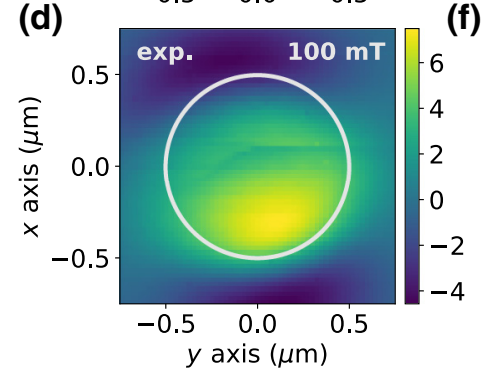

(b)

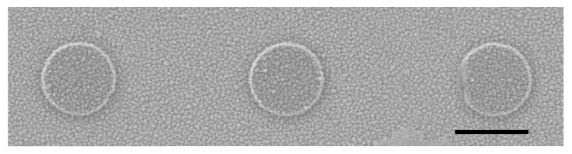

(g)

e)
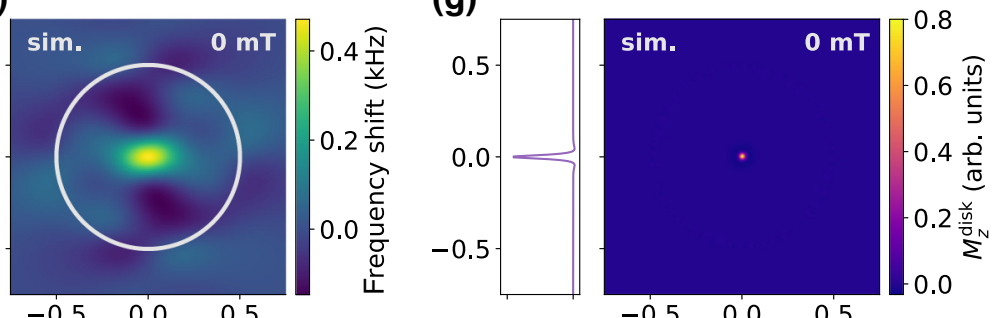

(h)
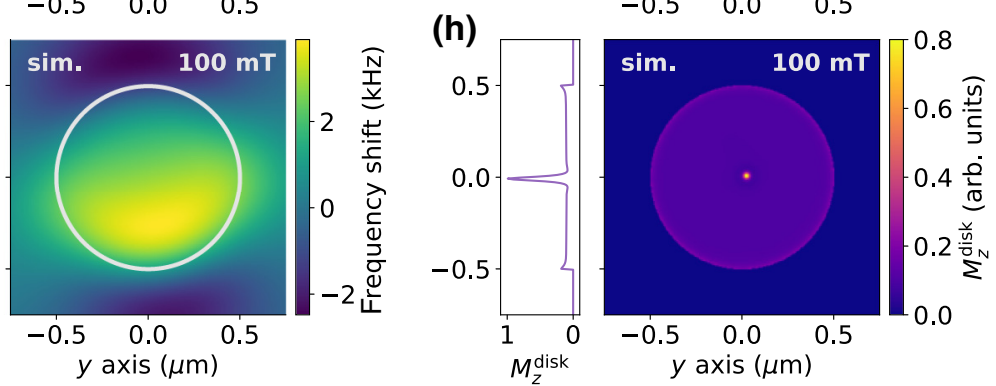

FIG. 4. (a) Sketch of the Au wire sample with three 1- $\mu \mathrm{m}$-wide permalloy disks patterned on top. The NW is driven resonantly by applying a gate voltage of $100 \mathrm{mV}_{p}$ amplitude at $f_{1}$. (b) SEM image of the permalloy disks. The scalebar is $1 \mu \mathrm{m}$. (c),(d) NW MFM images using NW 4 with an external magnetic field applied perpendicular to the disk plane of $B=0$ and $B=100 \mathrm{mT}$, respectively. (e),(f) Corresponding simulations of the expected contrast obtained by applying the monopole model (2) to the disk's stray field calculated by Mumax3. The disk circumference is highlighted by a white circle. (g),(h) Out-of-plane magnetization $M_{z}^{\text {disk }}$ of the permalloy disk simulated with Mumax for external magnetic fields of zero and $100 \mathrm{mT}$, and corresponding line cuts through the vortex core on the left hand side. The slight offset of the vortex core at nonzero external field is due a small tilt of the disk with respect to the $B$-field direction, which is taken into account for the simulation.

shift of the NW mode is proportional to the stray field derivative along the mode direction. While the contrast measured at the edges of the disk is due to topographic features, the contrast in the center is consistent with what is expected from the stray field of a vortex core. The image taken at $B=100 \mathrm{mT}$, Fig. 4(d), shows an almost homogeneous magnetic imaging contrast across the disk. The corresponding simulation in Fig. 4(f) agrees well with the measurement and reveals that while the vortex core is still present in the center of the disk, its stray field is overshadowed by the field originating from the outer parts of the disk, where the magnetization tilts out of plane [see Figs. 4(g) and 4(h)]. The region of high-frequency shift in the bottom-right quadrant of Fig. 4(d) is explained in the simulation by assuming a small tilt (approximately equal to $1^{\circ}$ ) of the disk plane with respect to the external field, resulting in the vortex core being slightly offset from the center of the disk. Although a detailed interpretation of the NW MFM images and a quantitative comparison of the measured and calculated frequency shifts is beyond the scope of this work, they showcase the high sensitivity and potential spatial resolution of the FEBID NW transducers.

\section{CONCLUSION}

In the past, FEBID-grown NWs have been patterned directly on tips of atomic force microscopy (AFM) cantilevers in an effort to improve spatial resolution [41, $44,45]$. Our results make clear that such nanocrystalline metallic NWs can have surprisingly high mechanical quality, making FEBID a promising and versatile method for producing nanometer-scale force transducers. In principle, a FEBID NW patterned on the tip of a standard AFM or MFM cantilever could be used to add sensitive 2D lateral force and dissipation detection capabilities. Such a system would be capable of vectorial force sensing in 3D, i.e., mapping both the size and the direction in 3D of tip-sample forces.

In addition to demonstrating the high-force sensitivity of FEBID-grown NWs, we also show their excellent magnetic properties. The Co NWs measured here maintain a 
saturation magnetization, which is $80 \%$ of the value of pure Co. They also have an axially aligned remanent magnetization with a switching field around $40 \mathrm{mT}$. These magnetic properties, combined with the aforementioned mechanical properties, make these NWs among the most sensitive sensors of local magnetic field. The ability to fine tune the NW geometry, especially making them thinner and sharper, may allow for even better field sensitivities and spatial resolutions in the future. NW MFM with such transducers may prove ideal for investigating subtle magnetization textures and current distributions on the nanometer scale, which - so far - have been inaccessible by other methods.

\section{ACKNOWLEDGMENTS}

We thank Sascha Martin and his team in the machine shop of the Physics Department at the University of Basel for help building the measurement system. We acknowledge the support of the Kanton Aargau, the ERC through Starting Grant NWScan (Grant No. 334767), the SNF under Grant No. 200020-178863, the Swiss Nanoscience Institute, and the NCCR Quantum Science and Technology (QSIT) as well as from the Spanish Ministry of Economy and Competitiveness (MINECO) through the projects MAT2017-82970-C1 and MAT2017-82970-C2 and from the Aragon Regional Government (Construyendo Europa desde Aragón) through project E13_20R, with European Social Fund funding. J.P.-N.'s grant is funded by the Ayuda para Contratos Predoctorales para la Formación de Doctores, Convocatoria Res. 05/06/15 (BOE 12/06/15) of the Secretaría de Estado de Investigación, Desarrollo e Innovación in the Subprograma Estatal de Formación of the Spanish Ministry of Economy and Competitiveness with the participation of the European Social Fund.

\section{APPENDIX A: INTERFEROMETRIC MEASUREMENT OF FLEXURAL MOTION}

We use a custom-built interferometer to detect the thermal or driven motion of the NW of interest. At its heart is a four-arm fiber coupler with a 95:5 coupling ratio. A Toptica $1550-\mathrm{nm}$ wavelength laser is connected to the input port and can be attenuated to the desired power. At the experiment arm of the fiber coupler (approximately equal to $5 \%$ transmission), an objective with a high numerical aperture focuses the laser light with a beam waist of around $1.65 \mu \mathrm{m}$ onto the NW. The interferometric displacement signal arises from the weak cavity between the NW and the end facet of the optical fiber. At the signal port of the fiber coupler the signal is converted to a voltage by a Femto OE300 photoreceiver and subsequently split into its dc and ac parts. Knowing the wavelength of the laser, the conversion factor between displacement and voltage can be determined accurately and is usually in the range of a few $\mu \mathrm{m} \mathrm{V} \mathrm{V}^{-1}$. A more detailed description of the optical setup can be found in the Supporting Information of Ref. [9].

The thermal displacement noise PSD, which is the projection of the motion of the two first-order flexural modes onto the direction of the local optical gradient, can be described by the fluctuation-dissipation theorem following the derivations in Ref. [10] as,

$$
\begin{aligned}
S_{x x}(\omega)= & u^{2}(z) \frac{4 k_{B} T}{m_{\mathrm{eff}}}\left[\frac{\omega_{1}}{Q_{1}} \frac{\cos ^{2} \alpha}{\left(\omega^{2}-\omega_{1}^{2}\right)^{2}+\left(\frac{\omega \omega_{1}}{Q_{1}}\right)^{2}}\right. \\
& \left.+\frac{\omega_{2}}{Q_{2}} \frac{\sin ^{2} \alpha}{\left(\omega^{2}-\omega_{2}^{2}\right)^{2}+\left(\frac{\omega \omega_{2}}{Q_{2}}\right)^{2}}\right]+S_{n},
\end{aligned}
$$

where $u(z)$ is the mode shape, $k_{B}$ the Boltzmann constant, $T_{\text {eff }}$ and $m_{\text {eff }}$ the effective temperature and mass of the NW resonator, $\omega_{1,2}$ and $Q_{1,2}$ the resonance frequencies and quality factors of the two first-order flexural modes, $\alpha$ the measurement angle between the direction of the first mode and the optical gradient, and $S_{n}$ the background noise. Depending on the position of the NW inside the beam waist, the optical gradient direction can be chosen at will. Ideally, however, it is aligned with the optical axis in order to achieve the best signal-to-noise ratio. The $z$ direction is aligned with the NW axis and its origin is located at the base of the NW. From the fit parameters, the spring constants $k_{1,2}=m_{\text {eff }} \omega_{1,2}^{2}$ and the thermally limited force sensitivity $F_{\min }=\sqrt{4 k_{B} T m_{\mathrm{eff}} \omega / Q}$ can be calculated.

\section{APPENDIX B: MICROMAGNETIC SIMULATIONS}

The principles of simulating the torque magnetometry signal with micromagnetic solvers are described in Refs. [9], [30], and [46]. In these works, it is only the tip of the mechanical resonator which is magnetic, therefore the system can be modeled as a magnetic object oscillating in a homogeneous external magnetic field. The mode shape of the mechanical resonator enters into the calculation only in the form of the effective length, simplifying the mechanics to that of a harmonic oscillator. For the Co NWs, which are both the mechanical resonator and the magnetic object, the mode shape has to be taken into account.

The final geometry used in the simulation of Fig. 2 is a 9.1- $\mu \mathrm{m}$-long elliptic cylinder, whose diameters are modulated along the $z$ direction, as determined from the SEM images. The average diameters along the two mode directions are $d_{1}=135 \mathrm{~nm}$ and $d_{2}=125 \mathrm{~nm}$. Space is discretized to $5 \mathrm{~nm}$. Material parameter values are the saturation magnetization $M_{\mathrm{sat}}=1.1(1) \times 10^{6} \mathrm{~A} \mathrm{~m}^{-1}$ and the exchange stiffness $A_{\mathrm{ex}}=38 \mathrm{pJ} \mathrm{m}^{-1}$. The latter has been chosen to match the switching field of the NW, and is significantly larger than other values reported for Co [47-50]. 

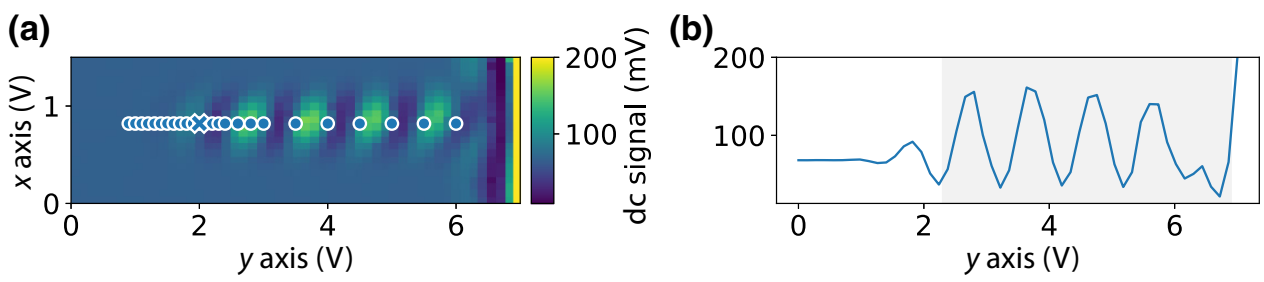

FIG. 5. (a) Scanning interferometer image of NW 4 at $T_{\text {bath }}=4.2 \mathrm{~K}$, with the spots marked at which the laser power dependence measurements are taken. The position marked by a cross (X) corresponds to the measurement position for the data shown in Figs. 6 and 7. Points correspond to measurement positions for data shown in Fig. 8. (b) Line scan along the long axis of the NW. The gray box highlights the extent of the NW. Interference fringes are visible in the images because the $z$-piezo scanner movement has a small spurious component along the $x$ direction.

This discrepancy arises because the switching field also depends sensitively on geometrical and material imperfections, which we do not attempt to model. Nevertheless, control simulations confirm that the overall magnetization reversal process and the remanent states are unaffected by such differences in $A_{\text {ex }}$.

In an effort to determine the effect of nanocrystallinity in the NW, we have run simulations with the NW divided into grains of around 10-nm size, giving each grain a uniaxial anisotropy with $K_{1}=530 \mathrm{~kJ} \mathrm{~m}^{-3}$ and a random orientation of the anisotropy axis. We find that this refinement does not significantly change the simulation results with respect to standard simulations assuming homogeneous material without crystalline anisotropy.

\section{APPENDIX C: BOLOMETRIC HEATING}

In order to investigate any bolometric heating of the NWs due to the readout laser, the thermal noise PSD is measured at the tip of NW 4 for laser powers from 0.5 to $25 \mu \mathrm{W}$ in room temperature $\left(T_{\text {bath }}=293 \mathrm{~K}\right)$, liquidnitrogen $\left(T_{\text {bath }}=77 \mathrm{~K}\right)$, and liquid-helium environments $\left(T_{\text {bath }}=4.2 \mathrm{~K}\right)$. In addition, the influence of the laser beam's position along the NW's long axis is examined for a fixed laser power of $20 \mu \mathrm{W}$. All laser powers provided in this section correspond to the total power at the output arm of the interferometer at the bottom of the probe, not the fraction of the power incident on the NW.
Generally, for high laser powers, a negative resonance frequency shift and a high thermal noise amplitude are observed, corresponding either to an increase in NW temperature or to a decrease in effective mass of the resonator. Since the latter can be excluded, we fix the effective mass to $2.6(2) \times 10^{-16} \mathrm{~kg}$, as extracted from the highest observed values at small incident laser powers with $T_{\text {bath }}=293 \mathrm{~K}$. The remaining data is then fit to Eq. (3) in the main text with the NW temperature $T=T_{\mathrm{NW}}$ as a free parameter.

The changes observed in the mechanical properties of the NW as a function of laser power and focal position are a consequence of the temperature dependence of the NW's Young's modulus [51]. A change of the Young's modulus is reflected in a change of a resonators resonance frequency, specifically in a negative frequency shift for higher NW temperatures.

\section{Laser power dependence}

Figure 5(a) shows an image of NW 4 with the positions marked, at which the thermal noise PSD is measured as a function of laser power while the bath is held at $T_{\text {bath }}=4.2 \mathrm{~K}$. Figure 5(b) shows a line cut of this image along the NW's long axis with the gray area highlighting the spatial extent of the NW. The NW's length is used to calibrate the conversion between piezo-scanner voltage and its displament in $\mu \mathrm{m}$. Resonance frequencies, quality factors, and NW temperatures of the first mode (a)

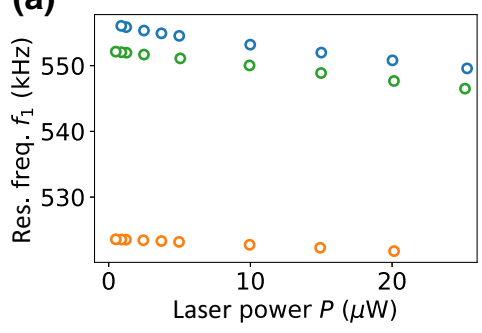

(b)

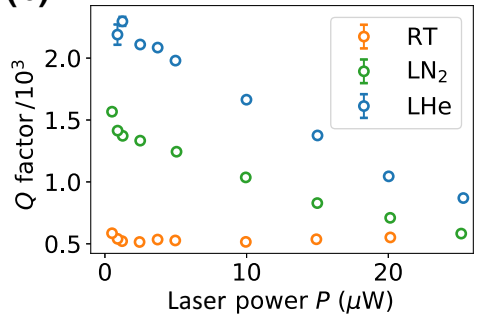

(c)

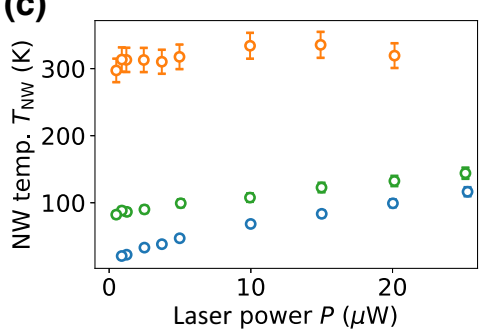

FIG. 6. Resonance frequency (a), quality factor (b), and NW temperature (c) at $T_{\text {bath }}=293 \mathrm{~K}$ (orange), $77 \mathrm{~K}$ (green), and $4.2 \mathrm{~K}$ (blue) for different laser powers. These power dependences are measured with the laser aligned to the tip of the NW 4, corresponding to the cross $(\mathrm{X})$ in Fig. 5(a). 

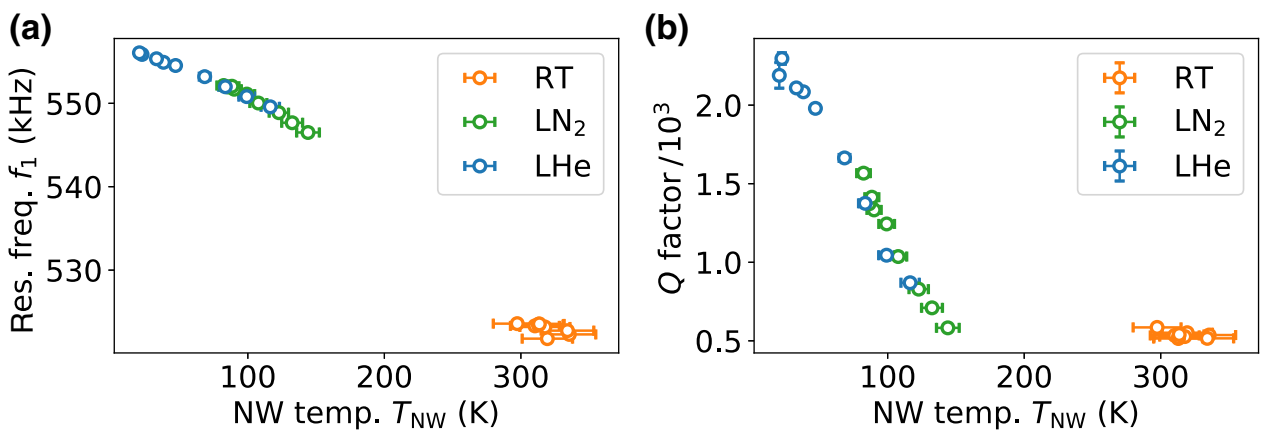

FIG. 7. (a) Resonance frequency of NW 4 plotted against the NW temperature for the full data set at all three different bath temperatures. The overlap of the points, especially at $T_{\text {bath }}=77 \mathrm{~K}$ and $4.2 \mathrm{~K}$, points to a bolometric heating effect. (b) Quality factor $Q$ against the NW temperature. These measurments are carried out with the laser aligned to the tip of the NW, corresponding to the cross (X) in Fig. 5(a).

are measured as a function of laser power at the position marked with the cross $(\mathrm{X})$ in Fig. 5 and plotted in Fig. 6.

For all three bath temperatures, the resonance frequency shows a linear decrease with increasing laser power. The quality factor exhibits a similar drop as a function of power for both $T_{\text {bath }}=4.2 \mathrm{~K}$ and $T_{\text {bath }}=77 \mathrm{~K}$, whereas at $T_{\text {bath }}=$ $293 \mathrm{~K}$ it appears constant within the error. The NW temperature increases with laser power at $T_{\text {bath }}=4.2 \mathrm{~K}$ and $T_{\text {bath }}=77 \mathrm{~K}$, while at $T_{\text {bath }}=293 \mathrm{~K}$, it appears roughly constant.

\section{Focal position dependence}

For a second set of measurements, the focal spot is moved along the $z$ axis (long axis) of the NW. Thermal noise PSDs are measured at the points shown in Fig. 5. Figure 8 shows the resonance frequency and the NW temperature along the NW, extracted from thermal noise PSDs, while taking into account the mode shape $u(z)$. As the focal spot is moved onto the NW tip and begins to overlap with the NW, the resonance frequency shifts downwards and the NW temperature rises, since maximal power impinges on the NW. At the other end of the NW, close to its base, the laser light is cut off by scattering at the chip edge. Consequently, the resonance frequency shifts up by a small amount and the NW temperature is lowered. Since the signal in this region is very small due to the mode's small displacement in this region and scattering at the chip edge, some measurement points for the NW temperature are omitted. (a)

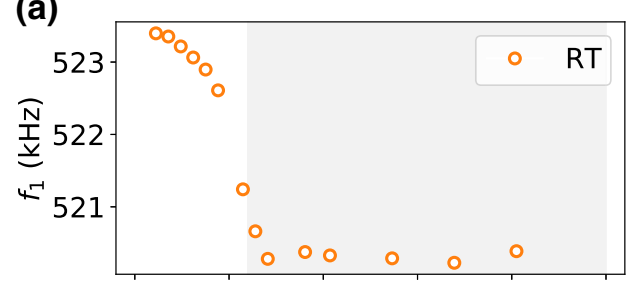

(c)
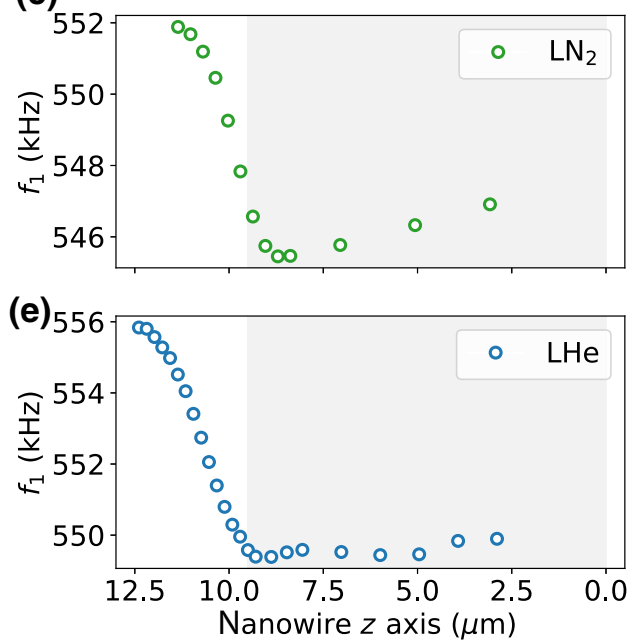

(b)

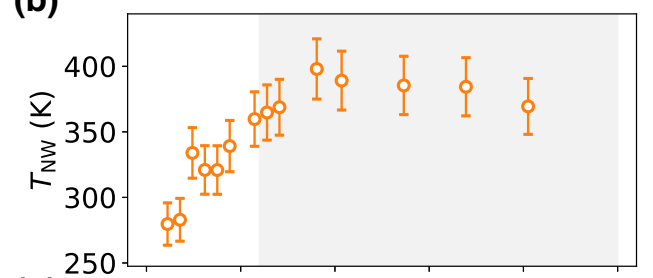

(d)
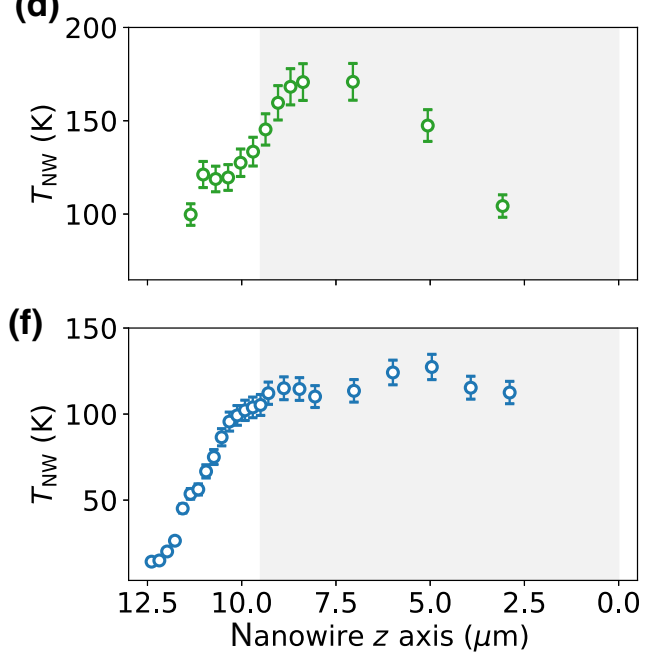

FIG. 8. (a)-(f) Resonance frequency and NW temperature at different points along the long axis of NW 4. The gray boxes mark the spatial extent of the NW. Moving the NW into the laser results in a lowering of the resonance frequency since the amount of incident power increases on the wire, thus heating it up. Once the NW is fully immersed in the laser beam, the NW temperature is roughly constant. The drop for the data at $T_{\text {bath }}=77 \mathrm{~K}$ in (d) can be explained by a small piezo drift perpendicular to the wire axis. Close to the base point the NW temperature decreases again due to a portion of the laser light being scattered at the chip edge. Note: the abscissa is inverted and its origin corresponds to the base point of the NW. 
In the middle region of the NW, where the laser focal spot fully overlaps with the NW, we measure a roughly constant NW temperature profile. This behavior is an indication that radiative losses - not thermal contact to the substrate - are the dominant channel through which the NW reaches thermal equilibrium. This observation indicates that the NW is well isolated thermally from the substrate and therefore, through the use of exchange gas to create thermal contact to a sample, could be used as a sensitive thermal scanning probe [52].

\section{APPENDIX D: BIOT-SAVART FIELD OF WIRE AND MONOPOLE MODEL}

The magnetic field of an infinitely long conductor with rectangular cross section of width $w$, height $h$, and a uniform current $I=j_{y} w h$ flowing in $y$ direction (see Fig. 9), where $j_{y}$ is the current density along the $y$ direction, can be calculated using the two-dimensional Biot-Savart law

$$
\begin{aligned}
& B_{x}(x, z)=\frac{\mu_{0} I}{2 \pi w h} \int_{s} \frac{z^{\prime}-z}{\left(x^{\prime}-x\right)^{2}+\left(z^{\prime}-z\right)^{2}} d S^{\prime}, \\
& B_{z}(x, z)=\frac{\mu_{0} I}{2 \pi w h} \int_{s} \frac{x^{\prime}-x}{\left(x^{\prime}-x\right)^{2}+\left(z^{\prime}-z\right)^{2}} d S^{\prime},
\end{aligned}
$$

where $S$ is its cross-sectional area. Solving the surface integrals with the center of the coordinate system aligned with the geometrical center of the conductor yields (see also Refs. [39] and [53])

$$
\begin{aligned}
B_{x}(x, z)= & \frac{\mu_{0} I}{4 \pi w h}\left[(w / 2-x) \log \frac{(w / 2-x)^{2}+(h / 2-z)^{2}}{(w / 2-x)^{2}+(h / 2+z)^{2}}\right. \\
& +(w / 2+x) \log \frac{(w / 2+x)^{2}+(h / 2-z)^{2}}{(w / 2+x)^{2}+(h / 2+z)^{2}} \\
& +2(h / 2-z)\left(\arctan \frac{w / 2-x}{h / 2-z}+\arctan \frac{w / 2+x}{h / 2-z}\right) \\
& \left.-2(h / 2+z)\left(\arctan \frac{w / 2-x}{h / 2+z}+\arctan \frac{w / 2+x}{h / 2+z}\right)\right]
\end{aligned}
$$

and a similar expression for $B_{z}(x, z)$.
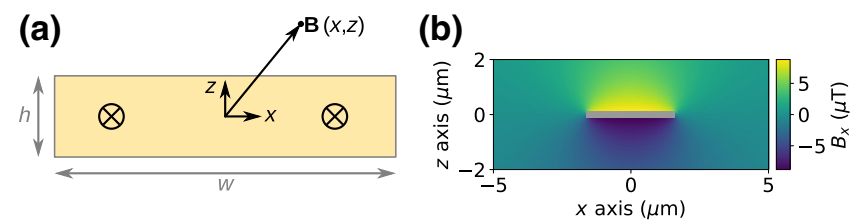

FIG. 9. (a) Cross section of an infinitely long rectangular conductor with width $w$ and height $h$. (b) Plot of $B_{x}(x, z)$ according to Eq. (D3) for $w=3.2 \mu \mathrm{m}, h=240 \mathrm{~nm}$ and a current of $I=47 \mu \mathrm{A}$.

In order to extract a value for the monopole $q_{0}$, the response of the NW to the driving tone is converted to a force using $F_{i}=r_{i} k_{i} / Q_{i}$ (i=1,2 representing the two modes). The interferometric calibration factor between units of signal amplitude in volts and displacement in meters and the measurement position along the wire axis have to be taken into account. We then use Eq. (D3) and the monopole model to fit the measured force response of NW 4 by assuming $\mathbf{B}_{\mathrm{ac}}(x, y, z)=\left[B_{x}(x, z), 0, B_{z}(x, z)\right]$ giving the fit function for the first mode

$$
F_{1}=q_{0} B_{x}(x, z) \cos \alpha^{\prime}
$$

where $\alpha^{\prime}$ is the angle between $x$ axis and the NW's first mode direction. It differs from $\alpha$ by the angle between the measurement direction and the optical axis, which is usually within the range of $2^{\circ}$ when centered on the wire. The optical axis is assumed to be aligned with the $x$ axis. The free parameters of the fit are the value of the monopole $q_{0}$ and its effective distance $z=z_{\text {mono }}$. A typical fit is shown in Fig. 3(c) of the main text for a distance between the tip and Au wire of $d_{z}=200 \mathrm{~nm}$ and a driving current of $47 \mu \mathrm{A}$. The field profile fitting to the response curve locates the effective monopole not at the tip of the NW, but higher along its axis at $z_{\text {mono }}=472(5) \mathrm{nm}$.

This behavior is illustrated in more detail in Fig. 10. The value of the monople is scattered, for different tipsample distances, in a region around 9.7(4) $\times 10^{-9} \mathrm{~A} \mathrm{~m}$. This spread can be related, on the one hand, to imperfections of the Au-wire structure resulting in a different (a)

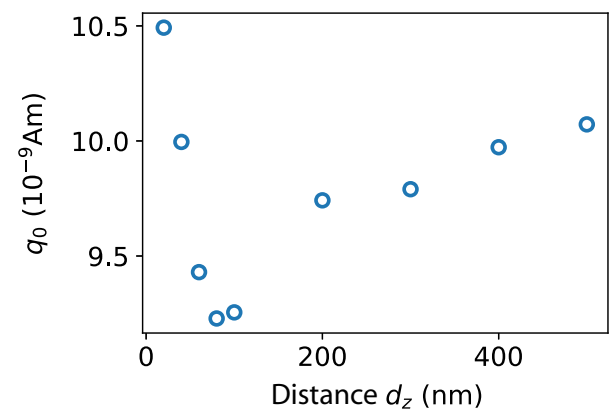

(b)

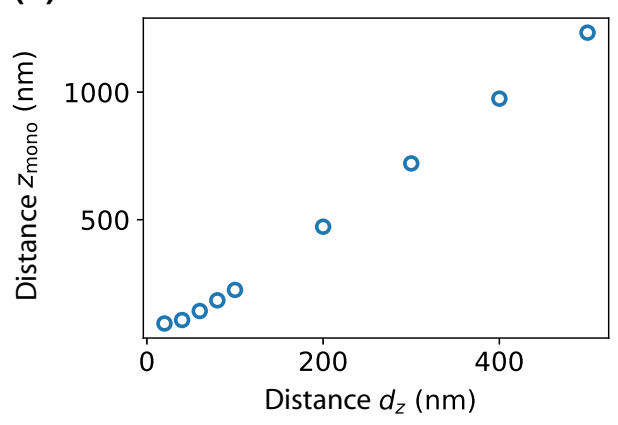

FIG. 10. (a) Value of the monopole $q_{0}$ and (b) monopole distance $z_{\text {mono }}$ plotted against the tip-sample distance $d$. The monopole distance increases with the tip-sample distance. The fitted value of the monopole is scattered around a value of 9.7(4) $\times 10^{-9}$ A m and seems to change substantially below a tip-sample distance closer than the NW's diameter. 
field profile and, on the other hand, to the approximate nature of the monopole model used for fitting the data. The tip of the NW, which interacts with the Biot-Savart field of the Au wire, is an extended object. Depending on the tip-sample distance $d_{z}$, its effective volume of interaction changes, leading to different values for $q_{0}$ and $z_{\text {mono }}$. In addition, a small tilt of the NW from the $x-y$ plane can also add error, since it introduces sensitivity to the $z$ component of $\mathbf{B}_{\mathrm{ac}}(x, y, z)$. It should also be noted that, while the zero point of the tip-sample distance is precisely determined by a soft touch of the NW tip onto the Au wire, the open-loop piezo translation stage regulating the tip-sample distance in $z$ direction is subject to piezoelectric creep. We do not consider the nonlinearity that creep introduces to the positioning of the NW. Furthermore, the nature of the open-loop scanners also introduces a systematic error of about $5 \%$ in the conversion of the $x$ and $y$ coordinates from voltage to $\mu \mathrm{m}$, depending on the calibration method used.

[1] X. Z. Yu, Y. Onose, N. Kanazawa, J. H. Park, J. H. Han, Y. Matsui, N. Nagaosa, and Y. Tokura, Real-space observation of a two-dimensional skyrmion crystal, Nature 465 , 901 (2010).

[2] K. C. Nowack, E. M. Spanton, M. Baenninger, M. König, J. R. Kirtley, B. Kalisky, C. Ames, P. Leubner, C. Brüne, H. Buhmann, L. W. Molenkamp, D. Goldhaber-Gordon, and K. A. Moler, Imaging currents in HgTe quantum wells in the quantum spin Hall regime, Nat. Mater. 12, 787 (2013).

[3] L. Thiel, Z. Wang, M. A. Tschudin, D. Rohner, I. GutiérrezLezama, N. Ubrig, M. Gibertini, E. Giannini, A. F. Morpurgo, and P. Maletinsky, Probing magnetism in $2 \mathrm{~d}$ materials at the nanoscale with single-spin microscopy, Science 346, 973 (2019).

[4] Ž. L. Jelić, A. Gurevich, A. V. Silhanek, E. O. Lachman, E. Zeldov, G. P. Mikitik, L. Embon, M. E. Huber, M. V. Milošević, Y. Anahory, and Y. Myasoedov, Imaging of super-fast dynamics and flow instabilities of superconducting vortices, Nat. Commun. 8, 85 (2017).

[5] A. Uri, Y. Kim, K. Bagani, C. K. Lewandowski, S. Grover, N. Auerbach, E. O. Lachman, Y. Myasoedov, T. Taniguchi, K. Watanabe, J. Smet, and E. Zeldov, arXiv:1908.02466 [cond-mat, physics:physics, physics:quant-ph] (2019).

[6] C. W. Hicks, L. Luan, K. A. Moler, E. Zeldov, and H. Shtrikman, Noise characteristics of $100 \mathrm{~nm}$ scale $\mathrm{GaAs} / \mathrm{Al}_{x} \mathrm{Ga}_{1-x} \mathrm{As}$ scanning Hall probes, Appl. Phys. Lett. 90, 133512 (2007).

[7] P. Maletinsky, S. Hong, M. S. Grinolds, B. Hausmann, M. D. Lukin, R. L. Walsworth, M. Loncar, and A. Yacoby, A robust scanning diamond sensor for nanoscale imaging with single nitrogen-vacancy centres, Nat. Nanotechnol. 7, 320 (2012).

[8] D. Vasyukov, Y. Anahory, L. Embon, D. Halbertal, J. Cuppens, L. Neeman, A. Finkler, Y. Segev, Y. Myasoedov, M. L. Rappaport, M. E. Huber, and E. Zeldov, A scanning superconducting quantum interference device with single electron spin sensitivity, Nat. Nanotechnol. 8, 639 (2013).

[9] N. Rossi, B. Gross, F. Dirnberger, D. Bougeard, and M. Poggio, Magnetic force sensing using a self-assembled nanowire, Nano Lett. 19, 930 (2019).

[10] F. R. Braakman and M. Poggio, Force sensing with nanowire cantilevers, Nanotechnology 30, 332001 (2019).

[11] S. J. Randolph, J. D. Fowlkes, and P. D. Rack, Focused, nanoscale electron-beam-induced deposition and etching, Crit. Rev. Solid State Mater. Sci. 31, 55 (2006).

[12] W. F. van Dorp and C. W. Hagen, A critical literature review of focused electron beam induced deposition, J. Appl. Phys. 104, 081301 (2008).

[13] I. Utke, P. Hoffmann, and J. Melngailis, Gas-assisted focused electron beam and ion beam processing and fabrication, J. Vac. Sci. Technol. B: Microelectron. Nanometer Struct. Process. Meas. Phenom. 26, 1197 (2008).

[14] M. Huth, F. Porrati, C. Schwalb, M. Winhold, R. Sachser, M. Dukic, J. Adams, and G. Fantner, Focused electron beam induced deposition: A perspective, Beilstein J. Nanotechnol. 3, 597 (2012).

[15] Nanofabrication Using Focused Ion and Electron Beams: Principles and Applications, edited by I. Utke, S. Moshkalev, and P. Russell (Oxford University Press, Oxford, New York, 2012).

[16] T. J. Gannon, G. Gu, J. D. Casey, C. Huynh, N. Bassom, and N. Antoniou, Focused ion beam induced deposition of low-resistivity copper material, J. Vac. Sci. Technol. B: Microelectron. Nanometer Struct. Process. Meas. Phenom. 22, 3000 (2004).

[17] H. Wu, L. A. Stern, D. Xia, D. Ferranti, B. Thompson, K. L. Klein, C. M. Gonzalez, and P. D. Rack, Focused helium ion beam deposited low resistivity cobalt metal lines with $10 \mathrm{~nm}$ resolution: Implications for advanced circuit editing, J. Mater. Sci.: Mater. Electron. 25, 587 (2014).

[18] J. M. De Teresa, A. Fernández-Pacheco, R. Córdoba, L. Serrano-Ramón, S. Sangiao, and M. R. Ibarra, Review of magnetic nanostructures grown by focused electron beam induced deposition (FEBID), J. Phys. D: Appl. Phys. 49, 243003 (2016).

[19] E. S. Sadki, S. Ooi, and K. Hirata, Focused-ion-beaminduced deposition of superconducting nanowires, Appl. Phys. Lett. 85, 6206 (2004).

[20] M. Esposito, V. Tasco, M. Cuscunà, F. Todisco, A. Benedetti, I. Tarantini, M. D. Giorgi, D. Sanvitto, and A. Passaseo, Nanoscale 3d chiral plasmonic helices with circular dichroism at visible frequencies, ACS Photonics 2, 105 (2015).

[21] T. Bret, T. Hofmann, and K. Edinger, Industrial perspective on focused electron beam-induced processes, Appl. Phys. A 117, 1607 (2014).

[22] L. A. Giannuzzi and F. A. Stevie, A review of focused ion beam milling techniques for TEM specimen preparation, Micron 30, 197 (1999).

[23] G. Nanda, E. van Veldhoven, D. Maas, H. Sadeghian, and P. F. A. Alkemade, Helium ion beam induced growth of hammerhead AFM probes, J. Vac. Sci. Technol. B 33, 06F503 (2015).

[24] C. H. Schwalb, C. Grimm, M. Baranowski, R. Sachser, F. Porrati, H. Reith, P. Das, J. Müller, F. Völklein, A. Kaya, 
and M. Huth, A tunable strain sensor using nanogranular metals, Sensors 10, 9847 (2010).

[25] R. Córdoba, A. Ibarra, D. Mailly, and J. M. De Teresa, Vertical growth of superconducting crystalline hollow nanowires by $\mathrm{He}^{+}$focused ion beam induced deposition, Nano Lett. 18, 1379 (2018).

[26] J. Pablo-Navarro, D. Sanz-Hernández, C. Magén, A. Fernández-Pacheco, and J. M. De Teresa, Tuning shape, composition and magnetization of $3 \mathrm{~d}$ cobalt nanowires grown by focused electron beam induced deposition (FEBID), J. Phys. D: Appl. Phys. 50, 18LT01 (2017).

[27] J. M. Nichol, E. R. Hemesath, L. J. Lauhon, and R. Budakian, Displacement detection of silicon nanowires by polarization-enhanced fiber-optic interferometry, Appl. Phys. Lett. 93, 193110 (2008).

[28] N. Rossi, F. R. Braakman, D. Cadeddu, D. Vasyukov, G. Tütüncüoglu, A. Fontcuberta i Morral, and M. Poggio, Vectorial scanning force microscopy using a nanowire sensor, Nat. Nanotechnol. 12, 150 (2017).

[29] D. Cadeddu, F. R. Braakman, G. Tütüncüoglu, F. Matteini, D. Rüffer, A. Fontcuberta i Morral, and M. Poggio, Timeresolved nonlinear coupling between orthogonal flexural modes of a pristine GaAs nanowire, Nano Lett. 16, 926 (2016).

[30] B. Gross, D. P. Weber, D. Rüffer, A. Buchter, F. Heimbach, A. Fontcuberta i Morral, D. Grundler, and M. Poggio, Dynamic cantilever magnetometry of individual $\mathrm{CoFeB}$ nanotubes, Phys. Rev. B 93, 064409 (2016).

[31] A. Vansteenkiste, J. Leliaert, M. Dvornik, M. Helsen, F. Garcia-Sanchez, and B. Van Waeyenberge, The design and verification of MuMax3, AIP Adv. 4, 107133 (2014).

[32] L. Exl, S. Bance, F. Reichel, T. Schrefl, H. Peter Stimming, and N. J. Mauser, LaBonte's method revisited: An effective steepest descent method for micromagnetic energy minimization, J. Appl. Phys. 115, 17D118 (2014).

[33] A. Mehlin, B. Gross, M. Wyss, T. Schefer, G. Tütüncüoglu, F. Heimbach, A. Fontcuberta i Morral, D. Grundler, and M. Poggio, Observation of end-vortex nucleation in individual ferromagnetic nanotubes, Phys. Rev. B 97, 134422 (2018).

[34] B. C. Stipe, H. J. Mamin, T. D. Stowe, T. W. Kenny, and D. Rugar, Magnetic Dissipation and Fluctuations in Individual Nanomagnets Measured by Ultrasensitive Cantilever Magnetometry, Phys. Rev. Lett. 86, 2874 (2001).

[35] M. J. Martínez-Pérez, J. Pablo-Navarro, B. Müller, R. Kleiner, C. Magén, D. Koelle, J. M. de Teresa, and J. Sesé, NanoSQUID magnetometry on individual As-grown and annealed Co nanowires at variable temperature, Nano Lett. 18, 7674 (2018).

[36] H. J. Hug, B. Stiefel, P. J. A. van Schendel, A. Moser, R. Hofer, S. Martin, H.-J. Güntherodt, S. Porthun, L. Abelmann, J. C. Lodder, G. Bochi, and R. C. O'Handley, Quantitative magnetic force microscopy on perpendicularly magnetized samples, J. Appl. Phys. 83, 5609 (1998).

[37] J. Lohau, S. Kirsch, A. Carl, G. Dumpich, and E. F. Wassermann, Quantitative determination of effective dipole and monopole moments of magnetic force microscopy tips, J. Appl. Phys. 86, 3410 (1999).

[38] P. J. A. van Schendel, H. J. Hug, B. Stiefel, S. Martin, and H.-J. Güntherodt, A method for the calibration of magnetic force microscopy tips, J. Appl. Phys. 88, 435 (2000).

[39] T. Kebe and A. Carl, Calibration of magnetic force microscopy tips by using nanoscale current-carrying parallel wires, J. Appl. Phys. 95, 775 (2004).

[40] Modern Techniques for Characterizing Magnetic Materials, edited by Y. Zhu (Springer, US, 2005).

[41] E. Berganza, M. Jaafar, M. Goiriena-Goikoetxea, J. PabloNavarro, A. Garcia-Arribas, K. Gusliyenko, C. Magen, J. M. de Teresa, O. Chubykalo-Fesenko, and A. Asenjo, Customized MFM probes based on magnetic nanorods, Nanoscale (2020).

[42] M. Poggio and C. L. Degen, Force-detected nuclear magnetic resonance: Recent advances and future challenges, Nanotechnology 21, 342001 (2010).

[43] R. Badea, J. A. Frey, and J. Berezovsky, Magneto-optical imaging of vortex domain deformation in pinning sites, J. Magn. Magn. Mater. 381, 463 (2015).

[44] I. Utke, P. Hoffmann, R. Berger, and L. Scandella, Highresolution magnetic Co supertips grown by a focused electron beam, Appl. Phys. Lett. 80, 4792 (2002).

[45] M. Stiller, J. Barzola-Quiquia, P. D. Esquinazi, S. Sangiao, J. M. De Teresa, J. Meijer, and B. Abel, Functionalized Akiyama tips for magnetic force microscopy measurements, Meas. Sci. Technol. 28, 125401 (2017).

[46] A. Mehlin, F. Xue, D. Liang, H. F. Du, M. J. Stolt, S. Jin, M. L. Tian, and M. Poggio, Stabilized Skyrmion phase detected in MnSi nanowires by dynamic cantilever magnetometry, Nano Lett. 15, 4839 (2015).

[47] G. Shirane, V. J. Minkiewicz, and R. Nathans, Spin waves in 3d metals, J. Appl. Phys. 39, 383 (1968).

[48] S. P. Vernon, S. Lindsay, and M. B. Stearns, Brillouin scattering from thermal magnons in a thin Co film, Phys. Rev. B 29, 4439 (1984).

[49] R. Krishnan, FMR studies in compositionally modulated Co-Nb and Co films, J. Magn. Magn. Mater. 50, 189 (1985).

[50] X. Liu, M. M. Steiner, R. Sooryakumar, G. A. Prinz, R. F. C. Farrow, and G. Harp, Exchange stiffness, magnetization, and spin waves in cubic and hexagonal phases of cobalt, Phys. Rev. B 53, 12166 (1996).

[51] E. Gil-Santos, D. Ramos, V. Pini, J. Llorens, M. FernándezRegúlez, M. Calleja, J. Tamayo, and A. S. Paulo, Optical back-action in silicon nanowire resonators: Bolometric versus radiation pressure effects, New J. Phys. 15, 035001 (2013).

[52] D. Halbertal, J. Cuppens, M. B. Shalom, L. Embon, N. Shadmi, Y. Anahory, H. R. Naren, J. Sarkar, A. Uri, Y. Ronen, Y. Myasoedov, L. S. Levitov, E. Joselevich, A. K. Geim, and E. Zeldov, Nanoscale thermal imaging of dissipation in quantum systems, Nature 539, 407 (2016).

[53] T. Göddenhenrich, H. Lemke, M. Mück, U. Hartmann, and C. Heiden, Probe calibration in magnetic force microscopy, Appl. Phys. Lett. 57, 2612 (1990). 